\title{
Reliability and validity of the Activities-specific Balance Confidence scale-Japanese (ABC-J) in community-dwelling stroke survivors
}

\author{
Satomi Ishige, MSc, PT ${ }^{1,2}$, Sawako WAKUI, $\mathrm{PhD}^{1}$, Yumi MiYaZAWA, $\mathrm{MD}^{3}$ and Hisashi NAITO, $\mathrm{PhD}^{1}$ \\ ${ }^{1)}$ Graduate School of Health and Sports Science, Juntendo University \\ ${ }^{2)}$ Department of Rehabilitation, Ushioda General Hospital \\ ${ }^{3)}$ Department of Neurology, Ushioda General Hospital
}

\begin{abstract}
Objective: In Japan, there were 1.17 million people with stroke in 2014; however, studies on community rehabilitation among stroke survivors are lacking. The Activities-specific Balance Confidence (ABC) scale is used in many languages to evaluate patients undergoing stroke rehabilitation. This study aimed to investigate the reliability and validity of the Japanese $\mathrm{ABC}$ scale (ABC-J) version among patients $\geq$ 6 months after stroke. Methods: This cross-sectional study was conducted with 88 post-stroke patients (mean age 66.5 \pm 9.5 years). The ABC-J was administered with the 10-meter walk test (10MWT), Timed Up and Go Test (TUG-T), Berg Balance Scale (BBS), Geriatric Depression Scale-Short version-Japanese (GDS-S-J), and the Falls Efficacy Scale-International (FES-I). After a 1-2-week interval, the ABC-J was completed again by 69 of the patients. Reliability was investigated for reproducibility (intra-class correlation coefficient [ICC], standard error of measurement [SEM], and minimal detectable change [MDC]) and internal consistency (Cronbach's $\alpha$ ). Concurrent and convergent validities were assessed using Spearman's rank correlation coefficients. Results: The ABC-J showed excellent internal consistency (Cronbach's $\alpha=0.95$ ) and substantial test-retest reliability (ICC $=\mathbf{0 . 9 2}, 95 \%$ confidence interval: $0.87-0.95$ ), with SEM and MDC of 7.14 and 19.79, respectively. The total ABC-J score was significantly correlated with $10 \mathrm{MWT}(\mathrm{r}=-\mathbf{0 . 5 1}, \mathrm{p}<0.001)$, TUG-T $(r=-0.55, p<0.001)$, BBS $(r=0.61, p<0.001)$, GDS-S-J $(r=-0.27, p=0.012)$, and FES-I $(r=-0.77$, p < 0.001). Conclusion: ABC-J is a valid and reliable measurement tool for investigating balance confidence among patients $\geq \mathbf{6}$ months after stroke.
\end{abstract}

Key words: balance ability, self-efficacy, activity daily living, fall, psychometric property

(Phys Ther Res 23: 15-22, 2020)

$\mathbf{P}_{\mathrm{r}}$ important for community rehabilitation in stroke survivors. The prevalence of falls after stroke is high and poor balance and mobility are particularly strong risk factors for falling among stroke survivors ${ }^{1)}$. PA in stroke survivors is generally low and most of their time is spent being inactive or sedentary ${ }^{2}$. Low PA after a stroke can lead to detrimental

Received: February 4, 2019

Accepted: July 18, 2019

Advance Publication by J-STAGE: October 10, 2019

Correspondence to: Sawako Wakui, Graduate School of Health and Sports Science, Juntendo University, 1-1 Hirakagakuendai, Inzai-city, Chiba 270-1695, Japan

\# e-mail: swakui@ juntendo.ac.jp

doi: 10.1298/ptr.E9982 secondary effects, such as stroke recurrence and onset of diabetes, hypertension, and/or dyslipidemia ${ }^{3)}$. In Japan, there were 1.17 million people with cerebrovascular disease in $2014^{4}$. However, studies on community rehabilitation among stroke survivors are lacking.

Self-efficacy (which is often understood to be selfconfidence) is a psychological concept that plays an important role in fall prevention and PA among stroke survivors. Self-efficacy is defined by an individual's belief in his or her innate ability to achieve set goals ${ }^{5)}$. According to systematic reviews on stroke rehabilitation, high self-efficacy is associated with enhancements in mobility, activities of daily living (ADL), and quality of life and reduced depression $^{6}$. Balance confidence is specifically associated with post-stroke $\mathrm{PA}^{7}$. 
Among several self-efficacy scales, the Activitiesspecific Balance Confidence (ABC) scale ${ }^{8)}$, originally developed in Canada by Powell and Myers, has been widely used in stroke studies. The ABC scale is used to assess balance confidence and comprises 16 situation-specific items related to indoor and outdoor activities (Appendix). The respondent indicates their level of self-efficacy at performing specific itemized activities without losing balance or becoming unsteady by choosing a corresponding number from $0 \%$ (no confidence) to $100 \%$ (completely confident). The validity and reliability of the $\mathrm{ABC}$ scale have been demonstrated in post-stroke survivors ${ }^{9-11}$. The $\mathrm{ABC}$ scale has been translated into many languages (ex. Chinese ${ }^{12,13)}$, Canadian-French ${ }^{9}$, and Swedish $\left.{ }^{10}\right)$ and validated by research in several countries ${ }^{14-16)}$.

A systematic review of PA interventions using balance self-efficacy measures in post-stroke survivors reported that almost all the studies (13 of 15) used the ABC scale as a secondary outcome ${ }^{17}$. The remaining two studies used the Falls Efficacy Scale International (FES-I), for which a Japanese version already exists, to assess the fear of falling ${ }^{17)}$. The $A B C$ scale has several characteristics: 1) it is used to measure subacute/chronic stage stroke rehabilitation, as recommended by the American Physical Therapy Association Neurology Section Task Force ${ }^{18} ; 2$ ) it can be used for international comparisons of balance confidence among post-stroke survivors; 3 ) it is easy to use because it comprises a simple questionnaire that can be completed in 5-20 minutes ${ }^{8}$; 4) it facilitates community stroke rehabilitation studies focused on improving ADL, preventing falls, and promoting PA; 5) it can be used to evaluate an individual's activity in the clinical setting. However, the ABC scale was not previously translated into the Japanese language, despite its many benefits. Therefore, the purpose of this study was to examine the reliability and validity of the Japanese version of the ABC scale (ABC-J) among community-dwelling stroke survivors.

\section{Method}

\section{Overview and study design}

This cross-sectional and test-retest designed study used a protocol that was based on the "Consensus-based Standards for the selection of Health status Measurement Instrument ${ }^{19),}$.

\section{Translation procedures}

Authorization and approval for translation were obtained from one of the original $\mathrm{ABC}$ scale authors ${ }^{8)}$. According to established international guideline ${ }^{20)}$, translation and cross-cultural adaptation of the $\mathrm{ABC}$ scale $^{8)}$ into Japanese was performed (Table 1). Briefly, the ABC scale was translated into Japanese by three researchers. A backtranslation was performed by native English translators and the scale was then pilot-tested in two stroke survivors. Some items required particularly careful translation, considering the Japanese culture and environment ${ }^{12-16)}$, which was performed in consultation with the original authors (Appendix). There were no issues with the translation of each item in the completed ABC-J regarding comprehensiveness and meaningfulness.

\section{Sample size estimation}

The sample size required to estimate reproducibility was calculated to be 50 based on an intra-class correlation coefficient $\left(\mathrm{ICC}_{2,1}\right)$ target value of 0.8 and a $95 \%$ confidence interval $(\mathrm{CI})$ of $\pm 0.1^{21)}$. The sample size required to estimate validity ${ }^{22)}$ was calculated to be 84 , according to Spearman's rank correlation coefficient, using an $\alpha$ of 0.05 , power of 0.80 , and medium effect size $(\rho)$ of $0.3^{9,11}$.

\section{Recruitment}

Post-stroke community-dwelling residents were recruited from daycare centers, rehabilitation home-visits, outpatient clinics, and medical fitness centers in the urban Kanagawa Prefecture. A research physiotherapist at each center identified the eligible patients via patient record reviews. The eligibility criteria were as follows ${ }^{9-11}$ : age 40-90 years; diagnosed by a neurologist with initial or recurrent stroke; $\geq 6$ months since the most recent stroke-onset; ability to walk 10 meters independently with or without a walking aid or supervision. The exclusion criteria comprised severe dysphasia and cognitive dysfunction with a limited ability to understand the test procedure.

\section{Procedure}

Eighty-eight participants completed the ABC-J and other measurement tools. These evaluations were completed by trained physical therapists at five facilities $(\mathrm{n}=$ 87). The evaluations of the medical fitness center participant $(n=1)$ were done by trained health fitness programmers under the supervision of a medical doctor. Sixty-nine of the participants completed the ABC-J after a 1-2-week interval. The participants filled out the ABC-J on their own, with the exception of those that had reading or writing difficulties secondary to hemiplegia or other reasons. All the participants completed the ABC-J either alone or with assistance. Information on sociodemographic variables [age, sex, body mass index, ADL: Barthel Index ${ }^{23}$, cognitive function by Mini Mental State Examination-Japanese $\left(\right.$ MMSE- $\left.^{24}\right)$ ] and stroke characteristics were obtained from medical records.

\section{Measurements}

ABC-J: the questionnaire contained 16 items scored on a range from $0 \%$ (no confidence) to $100 \%$ (completely confident). Scores were summed and divided by 16 , for a total score ranging from $0 \%$ to $100 \%$. 
Table 1. Translation procedure

(1) Preparation

Permission for translation was obtained from the original author.

(2) Forward Translation

Three native Japanese-speaking translators (an academic expert in behavioral science, a physiotherapist, and a neurologist) translated the ABC scale into Japanese. The translations included expressions that are normally used in the Japanese language so that the respondents could easily understand; however, significant efforts were made to avoid changing the meaning of the original $\mathrm{ABC}$ scale. After consulting with the original authors, some items were modified to include expressions that are specific to the culture of the original version, but expressed in accordance with the Japanese culture.

(3) Reconciliation

The preliminary version of the ABC-J was created after comparing and merging some of the forward translations into a single forward translation by three researchers.

(4) Back Translation

Native English translators who were not familiar with the original ABC scale and were not engaged in forward-translation made the translations back to the original language.

(5) Back Translation Review

To evaluate the quality of the forward translation, the back-translation version and the original $\mathrm{ABC}$ scale were compared and the original author was asked whether the two were equivalent or not. As a result of that review, no particular issues were identified.

(6) Harmonization

Comparison of the back-translations of 10 multiple-language versions with the original ABC scale to highlight discrepancies between the original version and the ABC-J, as well as to maintain a consistent approach to translation problems.

(7) Cognitive Debriefing

The translated ABC-J was pilot tested in two people (a women in her 40s and a man in his 60s) with stroke, both of whom were native Japanese speakers, to evaluate alternative wording and check comprehension, interpretation, and cultural relevance of the translation. There were no problems with any of the items when they were asked to comment on the comprehensiveness and meaningfulness of each question.

(8) Review of Cognitive Debriefing Results and Finalization

Two researchers (an academic expert in behavioral science and a physiotherapist) confirmed the results of part (7).

(9) Proofreading

A final review of the ABC-J was conducted to highlight and correct any typographical, grammatical or other errors.

(10) Final Report

Note. Created based on ISPOR task force 20 .

The following validation measures were selected based on previous studies ${ }^{9-11)}$ :

10 -meter Walk Test $(10 \mathrm{MWT})^{25)}$ : the $10 \mathrm{MWT}$ measures walking ability. Each participant walked 10 meters (with a 2-m acceleration path and a 2-m deceleration path) quickly, but safely. Two trials were performed, and the faster time was recorded.

Timed up \& Go Test (TUG-T) ${ }^{26)}$ : the TUG-T is an assessment of functional mobility that measures the time required to stand up from a chair, walk 3-m, turn around a corner, walk back, and sit back down. Two trials were performed and the faster time was recorded.

Berg Balance Scale (BBS) ${ }^{27)}$ : the BBS measures functional balance ability and comprises 14-items. Each item is scored in 5 stages from $0-4$ points. A higher point total (056 points) indicates greater balance ability.

Geriatric Depression Scale-Short version-Japanese $(G D S-S-J)^{28)}$ : the GDS-S-J is a depression assessment scale (originally the GDS-S ${ }^{29)}$ ) comprising 15-items answered "Yes" or "No". A higher total score (0-15 points) indicates more depressive symptoms.

Falls Efficacy Scale-International (FES-I $)^{30,31)}$ : the FES-I is a 16-item questionnaire indicative of fall-related self-efficacy that is rated with reference to "how concerned you are about the possibility of falling" during indoor and outdoor activities. Participants indicated their responses as follows (score in parentheses): not at all (1), somewhat (2), fairly (3), and very concerned (4). A higher score (16-64 points) indicates lower self-efficacy.

\section{Ethical approval}

We conducted this study upon approval of the institutional review boards (JUGSSHSREC, 28-3; UGHREC, 2015-03). Informed consent was obtained from each participant.

\section{Statistical analysis}

SPSS for Mac (ver. 24.0, IBM Corporation, Armonk, NY, USA) and Excel for Mac (ver. 16.16.2, Microsoft Corporation, Redmond, WA, USA) were used for the statistical analysis. Statistical significance was set at $\mathrm{p}<0.05$. 


\section{(1) ABC-J score distribution}

The mean score, standard deviation, median score, score range, and skewness/kurtosis were calculated to confirm acceptability. Acceptability was judged to be adequate when the observed scores were well-distributed, the mean scores were near the scale mid-point, the skewness/kurtosis statistics ranged between $-2 /+2^{32)}$, and the ceiling/floor effects were $<20 \%{ }^{33)}$. To assess ceiling/floor effects, the percentage of subjects who selected the minimum/maximum scores $(0,100$, respectively) was analyzed.

\section{(2) Reliability of the $A B C-J$}

Internal consistency was assessed using Cronbach's coefficient $\alpha$. Cronbach's $\alpha$ is regarded as excellent or good when $\alpha$ is $>0.9$ or $0.7-0.9$, respectively ${ }^{34)}$. To determine the relative reproducibility, the $\mathrm{ICC}_{2,1}$ was calculated. The $\mathrm{ICC}_{2,1}$ value was interpreted ${ }^{35)}$ as moderate (0.5-0.75), good (0.75-0.90), or excellent (>0.90). To assess absolute reliability, the standard error of measurement (SEM) and the minimal detectable change (MDC) were calculated ${ }^{36)}$.

(3) Concurrent and convergent validity of the ABC-J

Concurrent validity (10 MWT, TUG-T, BBS, and GDS-S-J) and convergent validity (FES-I) were analyzed using Spearman's rank correlation coefficient to determine the correlation strength between the total ABC-J score and the validation measures. The magnitude of significant coefficients (r) was evaluated as follows: small (0.1-0.29), medium (0.3-0.5), and large or strong $(\geq 0.5)^{37)}$.

\section{Results}

\section{(1) Demographic and functional data of the participants}

The demographic and functional results are shown in Table 2. The mean age of the 88 participants was $66.5 \pm 9.5$ years $($ men/women $=58 / 30)$ and the mean stroke interval was $4.8 \pm 4.2$ years. Seventy-nine $(89.8 \%)$ participants walked independently with or without canes or orthotic devices. Almost all participants were independent in ADL and had high cognitive function (mean BI and MMSE-J scores were $95.9 \pm 7.9$ and $28.2 \pm 2.8$, respectively).

(2) Descriptive statistics, internal consistency reliability, reproducibility, and measurement error of the $A B C-J$

Table 3 shows the following data: the mean ABC-J score was $57.5 \pm 24.1$, with a median value of 58 and a full range of responses to all items was observed. The skewness/kurtosis values of the $\mathrm{ABC}$ total and individual item scores were between -1.47 and +1.53 . There were no ceiling/floor effects in the ABC-J total score, whereas in the individual ABC-J items, a ceiling effect was observed in 8 and floor effect was observed in 4. Cronbach's $\alpha$ for the ABC-J was 0.95 and the ICC for test-retest reliability was 0.92 (95\% CI: $0.87,0.95)$. The SEM of the total ABC-J score was 7.14, with each item ranging from 11.16-17.88. The MDC individual item scores in the ABC-J ranged from
30.93-49.56 and the MDC total score was 19.79 (Table 3).

\section{(3) Concurrent and convergent validities of the ABC-J}

The results of the concurrent and convergent validities are shown in Table 4. The total ABC-J score was significantly correlated with the 10MWT $(\mathrm{r}=-0.51,95 \% \mathrm{CI}$ : $-0.65,-0.33, \mathrm{p}<0.001)$, TUG-T $(\mathrm{r}=-0.55,95 \% \mathrm{CI}$ : $-0.68,-0.37, \mathrm{p}<0.001)$, BBS $(\mathrm{r}=0.61,95 \% \mathrm{CI}: 0.45$, $0.72, \mathrm{p}<0.001)$, GDS-S-J $(\mathrm{r}=-0.27,95 \% \mathrm{CI}:-0.45$, $-0.06, \mathrm{p}=0.012)$, and FES-I $(\mathrm{r}=-0.77,95 \% \mathrm{CI}:-0.84$, $-0.66, \mathrm{p}<0.001)$.

\section{Discussion}

To the best of our knowledge, this is the first published study to evaluate psychometric properties of the ABC-J scale (translated based on international guidelines ${ }^{19,20)}$ ) among stroke survivors. The ABC scale is easily used and has notable value in clinical situations. Only reformulations were carried out, modifying some terms aimed at a better socio-cultural adaptation. Our descriptive statistics confirmed that the ABC-J is an acceptable scale for use in Japanese stroke survivors. Our results also showed evidence of excellent relative reliability and clinically acceptable absolute reliability.

The mean total ABC-J score in our study was similar to that of a previous post-stroke study (English and Canadian-French version $)^{9)}$ and was close to the median value. All values of skewness/kurtosis were within published criteria ${ }^{32}$. There were no ceiling/floor effects in total ABC-J score, whereas in the individual items, 8 ceiling and 4 floor effects were observed, respectively. In a previous post-stroke study ${ }^{9}$, nearly identical results were reported, so caution must be taken when evaluating individual items.

Cronbach's $\alpha$ of the ABC-J showed excellent internal consistency and was similar to those reported in post-stroke (English $^{9,11)}$, Canadian-French ${ }^{9)}$, and Swedish ${ }^{10)}$ ), Parkinson's disease $\left(\right.$ English $^{38,39)}$ and Italian ${ }^{15)}$ ), and communitydwelling elderly individuals or adults (English ${ }^{8,40)}$, Chinese $^{12)}$, and British English ${ }^{14)}$ ), where values ranged from 0.92 to 0.98 . The ICC of the total ABC-J also indicated excellent reliability ${ }^{35)}$. Although our value was superior to those reported in post-stroke (English ${ }^{11)}$ and Swedish ${ }^{10)}$ ), Parkinson's disease (English ${ }^{39)}$ ), and community-dwelling elderly individuals or adults (British English ${ }^{14}$ and Chinese ${ }^{12)}$ ), where values ranged from 0.79 to 0.89 , it was similar to that of Parkinson's disease (English ${ }^{38)}$ ), multiple sclerosis $\left(\right.$ English $\left.^{41)}\right)$, lower-limb amputation $\left(\right.$ English $\left.^{42)}\right)$, and community-dwelling elderly individuals (English ${ }^{8)}$ and Brazilian-Portuguese ${ }^{16)}$ ), where values ranged from 0.91 to 0.94. The SEM value in the current study was also similar to that of post-stroke studies (English ${ }^{9)}$, Canadian-French ${ }^{9}$, and Swedish ${ }^{11)}$ ); however, the MDC value in the current study was slightly greater than that of previous studies on 
Table 2. Demographic and functional data of the participants $(\mathrm{n}=88)$

\begin{tabular}{lcccc}
\hline \multicolumn{1}{c}{ Variable or Measure } & $\mathrm{n}$ & $\mathrm{M} \pm \mathrm{SD}$ & $\mathrm{MD}$ & (IQR) \\
\hline Age (years) & 88 & $66.5 \pm 9.5$ & 67 & $(61-73)$ \\
BMI $\left(\mathrm{kg} / \mathrm{m}^{2}\right.$ ) & 82 & $24.3 \pm 3.9$ & 23.9 & $(22.0-25.9)$ \\
Since stroke (years) & 88 & $4.8 \pm 4.2$ & 4.3 & $(1.6-6.8)$ \\
$\quad$ years: $<1, \geq 1$ & 9,79 & & & \\
Side of hemiplegia: & & & & \\
$\quad$ Right, Left, Bilateral & $46,38,4$ & & & \\
Type of stroke: IS, HS, Others & $45,42,1$ & & & \\
No. of strokes: 1, >1, unknown & $78,9,1$ & & & \\
Walking aid & & & & \\
$\quad$ Unaided, Aided & 46,42 & & & \\
$\quad$ Aided details: & & & & \\
$\quad$ TC, AFO & 10,8 & & & \\
$\quad$ TC + AFO, QC + AFO, WW + AFO & $19,2,1$ & & & \\
$\quad$ QC, PW & 1,1 & & & \\
Ambulation categories: IW, WS, WA & $79,4,5$ & & & \\
BI (score) & 88 & $95.9 \pm 7.9$ & 100 & $(95-100)$ \\
MMSE-J (score) & 87 & $28.2 \pm 2.8$ & 30 & $(27.5-30.0)$ \\
10MWT (sec) & 83 & $14.0 \pm 12.7$ & 9.9 & $(7.6-13.6)$ \\
TUG-T (sec) & 84 & $16.8 \pm 13.0$ & 11.8 & $(9.4-20.3)$ \\
BBS (score) & 86 & $45.8 \pm 9.1$ & 48 & $(42.0-52.0)$ \\
GDS-S-J (score) & 87 & $6.2 \pm 3.7$ & 6 & $(3.5-9.0)$ \\
FES-I (score) & 86 & $41.4 \pm 12.0$ & 42 & $(33.3-51.0)$ \\
\hline
\end{tabular}

Note. a All subjects had Barthel's lndex scores of 60 points or more (the amount of assistance was small). ${ }^{\mathrm{b}}$ Only one subject was unable to achieve a score of 25 , which was the cutoff for declining cognitive function.

Abbreviations. n: number of subjects; M: mean; SD: standard deviation; MD: median; IQR: interquartile range; BMI: body mass index; IS: ischemic stroke; HS: hemorrhagic stroke; TC: Tcanes; AFO: ankle-foot orthosis; WW: wheeled walker; QC: quad-canes; PW: pick-up walker; IW: independent walking; WS: watching support walking; WA: walking with assistance; BI: Barthel Index; MMSE-J: Mini-Mental State Examination-Japanese; 10MWT: 10m Walking Test; TUG-T: Timed up and Go Test; BBS: Berg Balance Scale; GDS-S-J: Geriatric Depression ScaleShort version-Japanese; FES-I: Falls Efficacy Scale-International.

patients with Parkinson disease (English ${ }^{38,39)}$ ). Thus, individual changes in ABC-J total scores of 0-19 points could be due to systematic or random errors and changes in total scores $\geq 20$ should be considered real, but not necessarily clinically relevant ${ }^{36)}$. Our absolute error suggests that the clinical measurement properties for the ABC-J were appropriate.

The validity of the ABC-J was judged to be adequate. As expected, strong negative correlations between the ABC-J and the 10MWT and TUG-T were consistent with previous post-stroke studies (English ${ }^{9,11)}$, Canadian-French"), and Swedish $^{10)}$ ). There was a strong positive correlation between ABC-J and BBS, and the correlation coefficient was larger than that of previous post-stroke studies (English ${ }^{9,11)}$ and Canadian-French ${ }^{9)}$ ). Although there are some differences in the strength of the correlation, our results support the link between balance confidence and actual physical function. The correlation between ABC-J and GDS-S-J was similar to that reported in previous studies $\left(\right.$ English $^{9,40)}$ and Canadian-French $^{9}$ ), which means that decreasing balance confidence is likely associated with depressive symptoms. Our strong negative correlation between ABC-J and FES-I was consistent with a previous study of vestibular disorders $^{43)}$, which demonstrates that the fear of falling is closely related to balance confidence.

This study has several methodological strengths. The main strength lies in the use of standardized and highly reliable and reasonable validation measures, which allowed us to perform adequate analyses. Our study provides valuable and high-quality data on mobility and physical function in stroke survivors. Our participants showed high physical and cognitive functioning as did participants in other countries $^{9-11)}$ and we were able to conduct our investigation as originally intended.

There are also some limitations in this study. First, our study population did not include subacute stroke survivors, 
Table 3. Descriptive statistics, internal consistency reliability, reproducibility, and measurement error of the ABC-J

\begin{tabular}{|c|c|c|c|c|c|c|c|c|c|c|c|c|c|c|}
\hline \multirow{3}{*}{$\begin{array}{l}\text { Item } \\
\text { numbera }\end{array}$} & \multicolumn{9}{|c|}{ Descriptive statistics and internal consistency reliability $(n=88)$} & \multicolumn{5}{|c|}{$\begin{array}{l}\text { Reproducibility and measurement } \\
\text { error }(n=69)\end{array}$} \\
\hline & \multirow{2}{*}{ M } & \multirow{2}{*}{ SD } & \multirow{2}{*}{ MD } & \multirow{2}{*}{ Range } & \multirow{2}{*}{ Sk } & \multirow{2}{*}{$\mathrm{Ku}$} & \multirow{2}{*}{$\mathrm{CE}$} & \multirow{2}{*}{$\mathrm{FE}$} & \multirow{2}{*}{$\alpha$} & \multirow{2}{*}{$\mathrm{ICC}^{\mathrm{c}}$} & \multicolumn{2}{|c|}{$95 \% \mathrm{CI}$} & \multirow{2}{*}{ SEM } & \multirow{2}{*}{ MDC } \\
\hline & & & & & & & & & & & $\mathrm{LL}$ & UL & & \\
\hline 8 & 79.7 & \pm 26.0 & 90 & $(0-100)$ & -1.47 & 1.53 & $42.0^{\mathrm{b}}$ & 2.3 & & 0.78 & 0.64 & 0.87 & 12.57 & 34.84 \\
\hline 9 & 77.7 & \pm 25.1 & 80 & $(0-100)$ & -1.10 & 0.64 & $39.8^{\mathrm{b}}$ & 1.1 & & 0.81 & 0.69 & 0.88 & 11.70 & 32.43 \\
\hline 4 & 76.3 & \pm 25.2 & 80 & $(0-100)$ & -1.24 & 1.35 & $33.0^{\mathrm{b}}$ & 3.4 & & 0.63 & 0.47 & 0.76 & 16.48 & 45.68 \\
\hline 10 & 75.3 & \pm 29.3 & 80 & $(0-100)$ & -1.19 & 0.38 & $37.5^{\mathrm{b}}$ & 3.4 & & 0.83 & 0.72 & 0.89 & 12.51 & 34.68 \\
\hline 1 & 74.4 & \pm 25.7 & 80 & $(0-100)$ & -1.00 & 0.41 & $29.5^{b}$ & 1.1 & & 0.80 & 0.68 & 0.87 & 11.73 & 32.51 \\
\hline 3 & 72.4 & \pm 27.0 & 80 & $(0-100)$ & -0.90 & -0.05 & $28.4^{b}$ & 2.3 & & 0.79 & 0.65 & 0.87 & 13.51 & 37.45 \\
\hline 14 & 64.2 & \pm 38.0 & 80 & $(0-100)$ & -0.73 & -1.04 & $31.8^{\mathrm{b}}$ & 18.2 & & 0.86 & 0.78 & 0.91 & 13.78 & 38.20 \\
\hline 11 & 60.7 & \pm 32.4 & 65 & $(0-100)$ & -0.43 & -0.99 & 19.3 & 8.0 & & 0.81 & 0.71 & 0.88 & 13.81 & 38.28 \\
\hline 2 & 59.7 & \pm 30.4 & 60 & $(0-100)$ & -0.38 & -0.76 & 18.2 & 8.0 & & 0.78 & 0.67 & 0.86 & 14.89 & 41.27 \\
\hline 7 & 52.8 & \pm 35.8 & 50 & $(0-100)$ & -0.14 & -1.28 & $21.6^{\mathrm{b}}$ & 18.2 & & 0.86 & 0.78 & 0.91 & 13.32 & 36.92 \\
\hline 5 & 49.8 & \pm 33.6 & 50 & $(0-100)$ & -0.05 & -1.22 & 12.5 & 15.9 & & 0.73 & 0.59 & 0.82 & 17.88 & 49.56 \\
\hline 12 & 48.6 & \pm 33.7 & 50 & $(0-100)$ & -0.03 & -1.27 & 10.2 & 17.0 & & 0.85 & 0.77 & 0.91 & 12.83 & 35.56 \\
\hline 13 & 37.5 & \pm 32.5 & 35 & $(0-100)$ & 0.34 & -1.13 & 5.7 & $27.3^{b}$ & & 0.86 & 0.79 & 0.91 & 12.51 & 34.68 \\
\hline 6 & 31.6 & \pm 35.5 & 20 & $(0-100)$ & 0.75 & -0.81 & 10.2 & $43.2^{\mathrm{b}}$ & & 0.88 & 0.81 & 0.92 & 12.42 & 34.43 \\
\hline 15 & 29.7 & \pm 34.9 & 15 & $(0-100)$ & 0.83 & -0.71 & 9.1 & $45.5^{\mathrm{b}}$ & & 0.90 & 0.84 & 0.94 & 11.16 & 30.93 \\
\hline 16 & 29.1 & \pm 32.2 & 20 & $(0-100)$ & 0.86 & -0.44 & 6.8 & $39.8^{\mathrm{b}}$ & & 0.86 & 0.78 & 0.91 & 12.45 & 34.51 \\
\hline $\begin{array}{l}\text { Total } \\
\text { ABC-J } \\
\text { score }\end{array}$ & 57.5 & \pm 24.1 & 58 & $(0-100)$ & -0.18 & -0.60 & 2.3 & 1.1 & 0.95 & 0.92 & 0.87 & 0.95 & 7.14 & 19.79 \\
\hline
\end{tabular}

Note. a Details are shown in the Appendix. Items are shown in high average value order. There are no missing data in any of the items. $\mathrm{b} \geq 20 \%$ ceiling or floor effect. $\mathrm{c}$ Two-way random effects absolute agreement.

Abbreviations. M: mean; SD: standard deviation; MD: median; Sk: skewness; Ku: kurtosis; CE: ceiling effects (\%); FE: floor effect (\%); $\alpha$ : Cronbach's alpha coefficient; ICC: the intraclass correlation coefficient; 95\%CI: 95\% confidence interval; LL: lower limit; UL: upper limit; SEM: standard error of measurement; MDC: minimum detectable change.

Table 4. Correlations between total ABC-J score and validation measures

\begin{tabular}{lrrrrr}
\hline \multirow{2}{*}{ Variable } & $\mathrm{r}$ & \multicolumn{2}{c}{$95 \% \mathrm{CI}$} & $\mathrm{p}$ \\
\cline { 3 - 4 } & & \multicolumn{1}{c}{$\mathrm{LL}$} & $\mathrm{UL}$ & \\
\hline 10MWT $^{\mathrm{a}}$ & -0.51 & -0.65 & -0.33 & $<0.001$ \\
TUG-T $^{\mathrm{b}}$ & -0.55 & -0.68 &,-0.37 & $<0.001$ \\
BBSc $^{\mathrm{n}}$ & 0.61 & 0.45 &, & 0.72 & $<0.001$ \\
GDS-S-Jd & -0.27 & -0.45 & -0.06 & 0.012 \\
FES-Ic & -0.77 & -0.84 & -0.66 & $<0.001$ \\
\hline
\end{tabular}

Note. a $\mathrm{N}=5$ missing, ${ }^{\mathrm{b}} \mathrm{N}=4$ missing, ${ }^{\mathrm{c}} \mathrm{N}=2$ missing, d $\mathrm{N}=1$ missing. p-value from Spearman's rank correlation analyses.

Abbreviations. r: Spearman's rank correlation coefficient; 95\%CI: 95\% confidence interval; LL: lower limit; UL: upper limit; 10MWT: 10m Walking Test; TUG-T: Timed Up and Go Test; BBS: Berg Balance Scale; GDS-S-J: Geriatric Depression Scale-Short version-Japanese; FES-I: Falls Efficacy Scale-International.

wheelchair users, or people with severe dysphasia or cognitive dysfunction. Second, this was a cross-sectional study; therefore, the predictive validity for fall prevention was not estimated. Third, we did not estimate PA. Although the balance confidence assessed by the $\mathrm{ABC}$ scale is considered to promote $\mathrm{PA}$, a follow-up study is necessary to examine the relationship between balance confidence and actual falls or PA levels to provide further evidence for predictive validity.

Despite these limitations, our study has some important implications. Although the ABC scale was developed for community elderly, according to the American Physical Therapy Association, the ABC scale was "recommended" as an outcome measure to assess not only patients with chronic stroke, but also those with subacute stroke and other neuromuscular diseases such as multiple sclerosis, Parkinson disease, and vestibular disorders. Therefore, the ABC-J can be used to evaluate community-dwelling elderly as well as patients with the aforementioned diseases. The ABC-J can also be used as an epidemiological study of Japanese stroke survivors in observational cross-sectional, case-control (e.g., between patients in Japan and those of other countries, between patients with stroke and those with other diseases, or among elderly patients living in a community), cohort, or experimental PA study designs. Finally, 
the ABC-J can be used as a clinical assessment tool to evaluate changes and improvements in personal selfefficacy.

\section{Conclusion}

The ABC-J is a valid and reliable measurement tool for investigating balance confidence among patients $\geq 6$ months after a stroke.

Acknowledgments: The authors would like to thank: Mr. S. Tanaka, PT, for recruiting the subjects and the staff of Ushioda General Hospital, S. Nishio, MD, Ph.D., and Mr. Y. Kuroda at Medical Corporation Unimedico Sunrise Family Clinic for assistance with the measurement assessments. This study was supported by the joint research program of Faculty of Health and Sports Science, Juntendo University and Institute of Health and Sports Science \& Medicine, Juntendo University.

Conflict of Interest: The authors affirm that they have no conflicts of interest related to this work.

\section{References}

1) Xu T, Clemson L, et al.: Risk factors for falls in community stroke survivors: a systematic review and meta-analysis. Arch Phys Med Rehabil. 2018; 99: 563-573.

2) Fini NA, Holland AE, et al:: How physically active are people following stroke? systematic review and quantitative synthesis. Phys Ther. 2017; 97: 707-717.

3) Billinger SA, Arena R, et al.: Physical activity and exercise recommendations for stroke survivors: a statement for healthcare professionals from the American Heart Association/American Stroke Association. Stroke. 2014; 45: 2532-2553.

4) Ministry of Health, Labour and Welfare [internet]: Tokyo: Trends in the estimated number of patients (per day) by type of medical institution and inpatients / outpatients, 1993-2014. [updated 2015 Dec. 17; 2014 Dec. 17]; Available from: https://ww w.mhlw.go.jp/toukei/list/10-20.html

5) Bandura A: Self-efficacy: toward a unifying theory of behavioral change. Psychol Rev. 1977; 84: 191-215.

6) Korpershoek C, van der Bijl J, et al.: Self-efficacy and its influence on recovery of patients with stroke: a systematic review. J Adv Nurs. 2011; 67: 1876-1894.

7) Thilarajah S, Mentiplay BF, et al.: Factors associated with poststroke physical activity: A systematic review and meta-analysis. Arch Phys Med Rehabil. 2018; 99: 1876-1889.

8) Powell LE and Myers AM: The activity-specific balance confidence (ABC) scale. J Gerontol A Biol Sci Med Sci. 1995; 50A: 28-34.

9) Salbach NM, Mayo NE, et al:: Psychometric evaluation of the original and Canadian French version of the Activities-specific Balance Confidence scale among people with stroke. Arch Phys Med Rehabil. 2006; 87: 1597-1604.
10) Forsberg $A$ and Nilsagård $Y$ : Validity and reliability of the Swedish version of the Activities-specific Balance Confidence scale in people with chronic stroke. Physiother Can. 2013; 65: 141-147.

11) Botner EM, Miller WC, et al.: Measurement properties of the Activities-specific Balance Confidence scale among individuals with stroke. Disabil Rehabil. 2005; 27: 156-163.

12) Hsu PC and Miller WC: Reliability of the Chinese version of the Activities-specific Balance Confidence scale. Disabil Rehabil. 2006; 28: 1287-1292

13) Mak MK, Lau AL, et al.: Validation of the Chinese translated Activities-Specific Balance Confidence scale. Arch Phys Med Rehabil. 2007; 88: 496-503.

14) Parry SW, Steen N, et al.: Falls and confidence related quality of life outcome measures in an older British cohort. Postgrad Med J. 2001; 77: 103-108.

15) Franchignoni F, Giordano A, et al.: Rasch validation of the Activities-specific Balance Confidence scale and its short versions in patients with Parkinson's disease. J Rehabil Med. 2014; 46: 532-539.

16) Marques AP, Mendes YC, et al.: Brazilian-Portuguese translation and cross cultural adaptation of the activities-specific balance confidence (ABC) scale. Braz J Phys Ther. 2013; 17: 170178.

17) Tang A, Tao A, et al.: The effect of interventions on balance self-efficacy in the stroke population: a systematic review and meta-analysis. Clin Rehabil. 2015; 29: 1168-1177.

18) Sullivan JE, Crowner BE, et al: : Outcome measures for individuals with stroke: process and recommendations from the American Physical Therapy Association neurology section task force. Phys Ther. 2013; 93: 1383-1396.

19) Mokkink LB, Terwee CB, et al:: The COSMIN checklist for assessing the methodological quality of studies on measurement properties of health status measurement instruments: an international Delphi study. Qual Life Res. 2010; 19: 539-549.

20) Wild D, Grove A, et al.: Principles of good practice for the translation and cultural adaptation process for patient-reported outcomes (PRO) measures: report of the ISPOR task force for translation and cultural adaptation. Value Health. 2005; 8: 94104

21) Giraudeau B and Mary JY: Planning a reproducibility study: how many subjects and how many replicates per subject for an expected width of the 95 per cent confidence interval of the intraclass correlation coefficient. Stat Med. 2001; 20: 3205-3214.

22) Faul F, Erdfelder E, et al.: Statistical power analyses using G* Power 3.1: Tests for correlation and regression analyses. Behav Res Methods. 2009; 41: 1149-1160.

23) Mahoney FI and Barthel DW: Functional evaluation: the Barthel Index. Md St Med J. 1965; 14: 61-65.

24) Sugishita M, Hemmi I, et al.: Reexamination of the validity and reliability of the Japanese version of the Mini-Mental State Examination (MMSE-J) Japanese. J Cogn Neurosci. 2016; 18: 3-4: 168-183. [Japanese].

25) Van Bloemendaal M, Van de Water AT, et al.: Walking tests for stroke survivors: a systematic review of their measurement prop- 
erties. Disabil Rehabil. 2012; 34: 2207-2221.

26) Podsiadlo D and Richardson S: The timed "Up \& Go": a test of basic functional mobility for frail elderly persons. J Am Geriatr Soc. 1991; 39: 142-148.

27) Berg K, Wood-Dauphinee S, et al.: Measuring balance in the elderly: preliminary development of an instrument. Physiother Can. 1989; 41: 304-311.

28) Sugishita K and Asada T: The creation of the Geriatric Depression Scale-Short version-Japanese (GDS-S-J). Jpn J Cognit Neurosci. 2009; 12: 87-90. [Japanese].

29) Sheikh JI and Yesavage JA: Geriatric Depression Scale (GDS). Recent evidence and development of a shorter version. Clin Geron. 1986; 5: 165-173.

30) Kamide N, Shiba Y, et al.: Reliability and validity of the Falls Efficacy Scale-International in Japanese community-dwelling elderly women. Sogo Rehabil. 2010; 38: 1063-1069. [Japanese].

31) Yardley L, Beyer N, et al.: Development and initial validation of the Falls Efficacy Scale-International (FES-I). Age Ageing. 2005; 34: 614-619.

32) George D and Mallery M: SPSS for Windows step by step: a simple guide and reference, 17.0 update. 10th ed, Pearson, Boston, 2010, pp. 21-22.

33) Hobart J and Cano S: Improving the evaluation of therapeutic interventions in multiple sclerosis: the role of new psychometric methods. Health Technol Assess. 2009; 13: 1-177.

34) Cronbach LJ: Coefficient alpha and the internal structure of tests. Psychometrika. 1951; 16: 297-333.

35) Koo TK and Li MY: A guideline of selecting and reporting intraclass correlation coefficients for reliability research. J Chiropr Med. 2016; 15: 155-163.

36) De Vet HCW, Terwee CB, et al.: Measurement in Medicine. In:
Practical guides to biostatistics and epidemiology, Cambridge University Press, Cambridge, 2011, pp. 111-112.

37) Cohen J: Statistical power analysis for the behavioral sciences. In: Routledge. 2nd ed, Lawrence Erlbaum Associates, Hillsdale, 1988, pp. 115-116.

38) Steffen $T$ and Seney M: Test-retest reliability and minimal detectable change on balance and ambulation tests, the 36-item short-form health survey, and the unified Parkinson disease rating scale in people with parkinsonism. Phys Ther. 2008; 88: 733-746.

39) Dal Bello-Haas V and Klassen L: Psychometric properties of activity, self-efficacy, and Quality-of-Life measures in individuals with Parkinson disease. Physiother Can. 2011; 63: 47-57.

40) Talley KM, Wyman JF, et al.: Psychometric properties of the Activities-Specific Balance Confidence scale and the survey of activities and fear of falling in older women. J Am Geriatr Soc. 2008; 56: 328-333.

41) Cattaneo D, Jonsdottir J, et al.: Reliability of four scales on balance disorders in person with multiple sclerosis. Disabil Rehabil. 2007; 29: 1920-1925.

42) Miller WC, Deathe AB, et al.: Psychometric properties of the Activities-specific Balance Confidence scale among individuals with a lower-limb amputation. Arch Phys Med Rehabil. 2003; 84: 656-661.

43) Morgan MT, Friscia LA, et al.: Reliability and validity of the Falls Efficacy Scale-International (FES-I) in individuals with dizziness and imbalance. Otol Neurotol. 2013; 34: 1104-1108.

\author{
Appendix \\ Original version of $\mathrm{ABC}$ scale
}

